São Paulo, São Paulo/SP, Brazil; '2 São Paulo/SP, São Paulo/SP, Brazil; ${ }^{3}$ Fundação Seade, São Paulo/SP, Brazil

Introduction High proportion of fetal autopsy is supposed to furnish information of better quality to death certificates.

Methods The completeness and underline cause of death was studied in a sample (212) death certificates from public system hospitals (539), 1st semester 2008, in São Paulo City, Brazil. This information was compared to hospital and coroner's office records and interviews were carried out with these professionals.

Results Public System Hospitals respond for $57.5 \%$ of all deliveries; $78.5 \%$ of fetal deaths were sent to coroners' office (CO) to be autopsied. The completeness of data of death certificates fulfilled by CO was worse than those emitted by hospitals. CO death certificates presented low completeness for mother's age (39.2\%); parity (29.9\%); type of gestation (3.9\%) and low concordance with hospital records. Gestational age (97.8\%) and birthweight $(99.3 \%)$ showed high completeness and were obtained from autopsy. Two causes of deaths respond up to $90 \%$ of $\mathrm{CO}$ death certificates: fetal death unspecified cause (65.8\%) and intrauterine hypoxia (24.3\%). The main causes from hospital certificates were: intrauterine hypoxia (41.7\%); fetus affected by maternal complications of pregnancy (19.4\%); fetal death unspecified cause (18.1\%).

Conclusions fetal deaths are mainly ante-partum and are understood as furnishing ill defined causes of deaths by obstetricians and were sent to autopsy. CO does not have accesses to hospital records, resulting in poor data quality. Instead of receiving social support to face fetal deaths, families were sent to police offices to get authorised file to transport fetus from hospitals to CO.

\section{SP6-16 THE ASSOCIATION OF BMI WITH SELF-RATED HEALTH AND FUNCTIONAL CAPACITY AMONG COMMUNITY-LIVING JAPANESE PEOPLE AGE OVER 75 YEARS}

doi:10.1136/jech.2011.142976p.87 people for promoting their health. Moreover, keeping good oral health is key element to prevent underweight among elderly people.

\section{SP6-17 SMOKING, LABOUR MARKET STATUS AND GENDER AMONG BRAZILIANS}

doi:10.1136/jech.2011.142976p.88

L Giatti, ${ }^{*}$ S Barreto. Federal University of Minas Gerais, Belo Horizonte, Minas Gerais, Brazil

Introduction Employment relations play an important role in explaining social inequalities in health.

Objective To investigated whether labour market status of men and women is associated with current smoking status, after adjusting for education, income and health and discuss hypotheses to explain the associations.

Methods Study included participants from National Health Survey of a nationally representative sample of Brazilians who were aged 15-64 years, economically active and residing in the eight metropolitan regions. Current smoking was defined as having a smoked at least 100 cigarettes in their life and who were currently smoking. Labour market status Associations were estimated by prevalence ratio and its $95 \%$ CI obtained by Poisson regression.

Results Among men, after all adjustments, workers without social protection and unemployed had higher prevalence of smoking (PR 1.31; 95\% CI 1.24 to 1.38 and PR 1.31; 95\% CI 1.18 to 1.44 respectively). Among women, corresponding figures were PR 1.22 (95\% CI 1.12 to 1.31) and PR 1.16 (95\% CI 1.03 to 1.32). The highest prevalence of smoking was found among male workers without social protection.

Conclusion Our results confirm that labour market status is an independent dimension of social inequalities related to smoke exposure. Despite being more pronounced among men, the social gradient was present in both genders. Future research should examine factors explaining this differences.

\section{SP6-18 UNEMPLOYMENT AND SELF RATED HEALTH: NEIGHBOURHOOD INFLUENCE?}

doi:10.1136/jech.2011.142976p.89 , hence approaching the risk of poor nutrition in the people is important. This study aimed to identify the associated factors of body mass index (BMI) among community-living Japanese people age over 75 years.

Methods A cross-sectional study was conducted using with a selfadministered questionnaire, and 658 participants (347 men and 331 women) were analysed. Demographic, lifestyle (eg, dietary habits, physical exercise), oral status, chronic illnesses, self-rated health, and activity of daily living (ADL) were asked. BMI was calculated from self-reported weight and height, and it was classified as underweight $(<18.4)$, normal weight (18.5-24.9), or obesity ( $\geq 25.0)$. Multiple logistic regression model were applied for analysis.

Results The study demonstrated that $70 \%$ of participants were normal weight, following to obesity $(21.4 \%)$, and underweight (8.5\%). Number of taking medicines, hypertension, diabetes, hyperlipaemia, and gastroenteropathy were associated with BMI. The adjusted $O R$ with age, gender, economic status, number of drugs being taken, and presence of associated illnesses for excellent/ good self-rated health tended to be lower among underweight participants $(O R=0.40, p=0.008)$, whereas higher among obese participants $(\mathrm{OR}=2.53, \mathrm{p}=0.001)$ than normal weight. In contrast, $\mathrm{ADL}$ was not associated with BMI. Compared to normal weight participants, underweight participants tended to perceive chewing problem $(\mathrm{OR}=2.47, \mathrm{p}=0.003)$, and not always eat 3 meals in a day $(\mathrm{OR}=3.75, \mathrm{p}=0.003)$.

Conclusion The results suggested that nutritional interventions should be targeted particular to underweight latter-stage elderly

L Giatti, * S Barreto, C César. Federal University of Minas Gerais, Belo Horizonte, Minas Gerais, Brazil

Objetive To investigated whether unemployment and socioeconomic characteristics of the neighbourhood are associated with poor self rated health after adjustment for individual sociodemographic characteristics, behavioural risk factors and health status. Moreover, it tests whether living in socioeconomic deprivation areas modifies the association between unemployment and self rated health.

Methods Participants aged 15-64 years, living in four Brazilian Capitals included in the National Household Survey on Risk Behaviours and Reported Morbidity from Non-Communicable Diseases (2002/2003). Data from the 2000 Brazilian Population Census were used for building up two socioeconomic neighbourhood indicators: the proportion of householders with low income, a compositional variable of individual level characteristics, and residing in slums, a contextual variable not captured by individuals properties. Logistic regression analysis was estimated by Generalised Estimating Equations.

Results From the 6426 participants, 20.6\% reported poor self-rated health. Unemployment as well as residing in slums or in low income household areas were significantly associated with poor self-rated health. The magnitudes of these associations were attenuated after 
adjustment for sociodemographic characteristics, behavioural risk factors and health condition of individuals. But, the association between unemployment and poor self-rated health was not modified by neighbourhood socioeconomic indicators.

Conclusion Results confirm the association between unemployment and poor self rated health, regardless the personal or contextual characteristics studied here. Similarly, they show a clear independent association between self rated health and neighbourhood context. But, they do not show that the neighbourhood contexts investigated modify the associations between unemployment and poor self rated health.

\section{SP6-19 SERUM CONCENTRATION OF HCH, DDT AND METABOLITES IN ADOLESCENTS EXPOSED TO ORGANOCHLORINE PESTICIDE RESIDUES IN BRAZIL}

doi:10.1136/jech.2011.142976p.90

R Guimarães, ${ }^{*}$ I Braga, A Vianna, C Asmus. Federal University of Rio de Janeiro, Institute of Studies in Public Health, Rio de Janeiro, Brazil

Objective To evaluate the concentration of biomarkers of exposure to residues of organochlorine pesticides in adolescents in Rio de Janeiro, Brazil

Methods We obtained laboratory results of serum concentrations of the isomers of $\mathrm{HCH}$ and DDT metabolites from the data collection from medical records at a local health unit from a previous screening.

Results We observed concentrations were considered well above the tolerance limits allowed. The distribution shows typical asymmetrical pattern, because of the presence of individuals with concentrations outliers distribution. Deserve special attention from the $\mathrm{HCH}$ congeners, the p,p'DDT and p,p'DDE; whose serum concentration mean and median are much higher than the level of tolerance permitted. Concentration obtained were from a $\mathrm{HCH}$ (115.81 Mean \pm SD 1438.529), b HCH (1616266 Mean \pm SD 135.15), g HCH (Mean \pm 5373.357 SD 447.66), p, p'DDE (Mean $94.92 \pm 884979$ SD), p, p 'DDD (5:23 Mean \pm 43350 SD), o, p 'DDT (3596 Mean $1.82 \pm \mathrm{SD}$ ) and p, p' DDT (45.61 Mean \pm SD 464389). In measuring the correlation between different types of organochlorine compounds evaluated, it is observed that the majority has mayor correlation $(r=1.000)$, or nearly so, with great statistical significance $(p<0.0001)$.

Conclusion There is needing for monitoring and periodic evaluation of health of this population, considering that the blood is altered especially at this critical period of development.

\section{SP6-20 OCCUPATIONAL HEALTH RISKS: RELIABILITY TEST FROM INGECTH-SUS STUDY}

doi:10.1136/jech.2011.142976p.91

${ }^{1} \mathrm{M}$ Y Mauro, ${ }^{2} \mathrm{R}$ Guimarães, ${ }^{*} \mathrm{~L}$ Santiago, ${ }^{4} \mathrm{C}$ Mauro. ${ }^{1}$ University of State of Rio de Janeiro, Rio de Janeiro, Brazil: ${ }^{2}$ Federal University of Rio de Janeiro, Institute of Studies in Public Health, Rio de Janeiro, Brazil; ${ }^{3}$ Oswaldo Cruz Foundation, Rio de Janeiro, Brazil; ${ }^{4}$ MI2 Ergonomics, Rio de Janeiro, Brazil

The goal of this study is to present the reliability test of Boix and Vogel's risk assessment scale in work environments for health area in Brazil. For this, 73 nursing professionals from Brazil answered the scale version, and replied the instrument with a range of 15 days. For internal consistency evaluation of dimensions it was used the Cronbach's $\alpha$; to assess the strength of association between the constructs was used the intraclass correlation coefficient (ICC); and to evaluate the consistency of response in two times interview was used the weighted $\kappa$. The ICC showed excellent correlation between dimensions, ranging from 0.87 and 0.96 . About internal dimensions consistency, Cronbach's $\alpha$ values varied from 0.64 to 0.85 . On the items, the weighted $\kappa$ varied from 0.66 (95\% CI 0.52 to 0.81 , and 0.93 ( $95 \%$ CI 0.87 to 0.99 ). The results of this study indicate that the scale shows high reproducibility for all dimensions evaluated.

\section{SP6-21 PATTERNS OF BREASTFEEDING AND DETERMINANT FACTORS IN BRAZIL}

doi:10.1136/jech.2011.142976p.92

${ }^{1} \mathrm{P}$ Pinto, ${ }^{2} \mathrm{R}$ Guimarães, ${ }^{*}{ }^{3} \mathrm{~L}$ Rangel, ${ }^{4} \mathrm{M}$ Ribeiro. ${ }^{1}$ Gaffrée Guinle University Hospital, Rio de Janeiro, Brazil; ${ }^{2}$ Federal University of Rio de Janeiro, Institute of Studies in Public Health, Rio de Janeiro, Brazil; ${ }^{3}$ Rio de Janeiro University, Rio de Janeiro, Brazil; ${ }^{4}$ Federal University of Rio de Janeiro, Rio de Janeiro, Brazil

Breastfeeding has innumerable advantages on the child, its family and society. Although the governmental efforts, of the professionals of health and the society in the direction to promote, to protect and to support this practical, the prevalence of exclusive breastfeeding until 6th life month remains less than ideal. The objective of this study is to determine the correlation of exclusive and non-exclusive breastfeeding with some basic health indicators, supplying data that they may be able to help for the development of interventions that include breast-feeding overall its conjuncture. An ecological study has been carried through using secondary data from DATASUS will be, from the period of 1999 to 2000's. The correlation has been esteemed through Pearson's coefficient. Is was observed a statistically significant correlation to variables related to marital status, education, coverage of prenatal, delivery characteristics and infant mortality rate after neonatal and proportional for IPD. It is observed therefore that the actions, especially in prenatal and multiplepatient room, must be prioritised in these groups which showed an inverse correlation.

\section{SP6-22 STUDY OF INFRASTRUCTURE AND MANPOWER OF PHCS AND SUB-CENTRES IN NELLORE DISTRICT, ANDHRAPRADESH, INDIA}

doi:10.1136/jech.2011.142976p.93

S Sriram, ${ }^{*}$ V Chandrasekhar. Narayana Medical College, Nellore, Andhrapradesh, India

India has a vast public health infrastructure in the country with 23391 primary health centres and 145894 sub-centres providing health services to $72.2 \%$ of the country's population living in rural areas. Although the numbers look impressive their functional status needs to be improved in terms of physical infrastructure, manpower, equipment, drugs and other logistic supply that are very much needed for ensuring quality services. National Rural Health Mission (NRHM) launched aims to restructure the delivery mechanism for health towards providing universal access to equitable, affordable and quality healthcare, as a result of which Indian Public Health Standards (IPHS) were formulated. The present work aims to study the infrastructure facilities and manpower in a sample of Primary Health Centres (PHCs) and their subcentres in the district of Nellore in the state of Andhra Pradesh in the east coast of India. A randomly selected sample of health institutions have been studied using structured and pre-tested performance. The data have been analysed with reference to Indian Public Health Standards (IPHS). It is a cross-sectional community based study done over a period of 1 year in Nellore district in the study setting of primary health centres and sub-centres. There are 62 primary health centres in Nellore district distributed in three divisions (Gudur, Nellore and Kavali). 25\% of the primary health centres have been selected randomly that is, 15 PHCs taking five from each division randomly. Each PHC consists of 